\title{
AVALIAÇÃO DA TOXICIDADE AGUDA PARA O ORGANISMO-TESTE Vibrio Fischeri DOS PRINCIPAIS HERBICIDAS E INSETICIDAS APLICADOS NA LAVOURA DE ARROZ IRRIGADO DOS ESTADOS DE SANTA CATARINA E RIO GRANDE DO SUL
}

\author{
FERNANDA POLEZA* \\ RAFAEL CAMARGO SOUZA** \\ CESAR AUGUSTO STRAMOSK*** \\ LEONARDO RUBI RORIG****

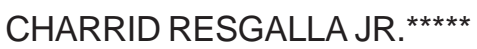

\begin{abstract}
Santa Catarina e Rio Grande do Sul são os maiores produtores de arroz irrigado do Brasil e destacam-se pelo uso intenso de pesticidas para o controle de competidores e consumidores indesejáveis. A fim de avaliar o potencial tóxico desses agroquímicos e seus efeitos sobre organismos não-alvo, 0 presente trabalho teve como objetivo determinar a $C_{20}$ e a $C_{50}$ dos principais herbicidas e inseticidas empregados na lavoura do arroz, utilizando como organismo-teste a bactéria bioluminescente Vibrio fischeri. Foram realizados testes estáticos de curta duração (30 minutos), baseados na inibição da luminescência das bactérias após exposição ao agente tóxico. Os produtos testados foram os herbicidas 2,4-D (DMA 806 BRdimetilamida), metsulfurom metílico (Ally), carfentrazona-etílica (Aurora), bentazona (Basagran), quincloraque (Facet), oxifluorfem (Goal), bispiribaque-sódico (Nominee) e pirazossulfuron etílico (Sírius), e os inseticidas betaciflutrim (bulldock), carbofuran (Furadan), lambdacialotrina (Karate) e fipronil (Standak) em concentrações máximas de 100 mg/L (i.a.). Os herbicidas Ally e Sírius e os inseticidas Bulldock e Standak não apresentaram toxicidade para a bactéria Vibrio fischeri, pois resultaram em efeito inibitório inferior a 20\%. Basagram, Facet, Nominee e Furadam apresentaram toxicidade, com valores de $\mathrm{CE}_{20}$ (i.a.) de 40,87 mg/L, $36 \mathrm{mg} / \mathrm{L}, 45,83 \mathrm{mg} / \mathrm{L}$ e $3,23 \mathrm{mg} / \mathrm{L}$, respectivamente. Para os produtos 2,4-D, Aurora, Goal e Karatê, os valores de $\mathrm{CE}_{50}$ dos ingredientes ativos foram 13,90 mg/L, 1,02 mg/L, 19,23 mg/L e $10 \mathrm{mg} / \mathrm{L}$, respectivamente. Em comparação com estudos realizados com outros organismos-teste, a bactéria $V$. fischeri e o teste de bioluminescência apresentaram baixa sensibilidade frente à toxicidade dos agroquímicos.
\end{abstract}

PALAVRAS-CHAVE: TESTES DE TOXICIDADE; HERBICIDAS; INSETICIDAS; BIOLUMINESCÊNCIA; Vibrio fischeri.

\footnotetext{
* Bióloga, Centro de Ciências Tecnológicas da Terra e do Mar (CTTMar), Universidade do Vale do Itajaí (UNIVALI), Itajaí, SC (e-mail: poleza@univali.br).

** Biólogo, CTTMAR, UNIVALI, Itajaí, SC (e-mail: rafael.souza@univali.br).

*** Oceanógrafo, CTTMAR, UNIVALI, Itajaí, SC (e-mail: stramosk@yahoo.com.br).

**** Doutor em Ecologia, CTTMAR, UNIVALI, Itajaí, SC (e-mail: rorig@univali.br).

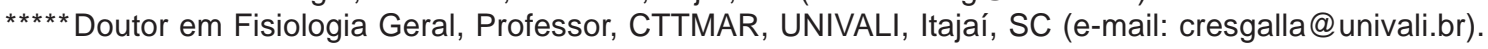




\section{INTRODUÇÃO}

O arroz irrigado ocupa 1,2 milhões de hectares nos estados do Rio Grande do Sul e Santa Catarina, que são responsáveis por quase $60 \%$ da produção nacional desse cereal (NOLDIN, 2005). Os sistemas de cultivo predominantes são a semeadura direta em solo drenado e principalmente 0 pré-germinado, caracterizado pela semeadura em lâmina de água (NOLDIN et al., 2001).

O intenso desenvolvimento da agricultura brasileira tem acentuado a utilização de agroquímicos e sua aplicação excessiva tem causado grande impacto ao meio ambiente, podendo provocar efeitos danosos à saúde humana.

Dependendo do tipo de manejo de água, adotado pelos produtores e das condições de precipitação pluviométrica após as aplicações, existe o risco dos resíduos dos agroquímicos serem carreados para fora da lavoura, afetando os organismos aquáticos nas águas a jusante (RESGALLA JR. et al., 2002).

Diversos estudos vêm sendo realizados com o intuito de avaliar o potencial tóxico dos agroquímicos no ambiente. DESCHAMPS et al. (2003) avaliaram a ocorrência de resíduos de agroquímicos em águas nas áreas de arroz irrigado em Santa Catarina. Outros estudos avaliaram a toxicidade dos agroquímicos sobre diferentes organismos como juvenis de carpa (RESGALLA JR. et al., 2002), o cladócero Daphnia magna (NAKAGOME, NOLDIN e RESGALLA, 2006), o peixe Danio rerio (NAKAGOME, 2004) e a microalga Pseudokirchneriella subcaptata (TAMANAHA et al., 2003). Todos esses estudos alertaram para o potencial tóxico que determinados herbicidas e inseticidas podem exercer sobre o ambiente, porém nem sempre seus resultados são concordantes.

É de fundamental importância a avaliação da toxicidade dos agroquímicos sobre organismos não-alvo, tornando-se necessária a procura por novos dados de sensibilidade com diferentes organismosteste para verificar o potencial tóxico e a seletividade dos compostos.

Entre a grande variedade de sistemas para medição da toxicidade, alguns métodos são caros, pouco seguros e não fornecem resposta rápida, além de promoverem objeções éticas com relação a utilização de animais (ALBA et al., 2001).

Os bioensaios com a bactéria bioluminescente Vibrio fischeri utilizando o pacote LUMISTOX ${ }^{\circledR}$ têm demonstrado vantagens como, simplicidade, rapidez e baixo custo. São baseados na inibição da emissão de luz dessas bactérias, após o período de contato com a substância-teste (STRAMOSK, 2004).

Vibrio fischeri é uma enterobacteria, Gram negativa, pertencente à família Vibrionaceae, constituída de muitas espécies que são caracterizadas pela cooperação e interação com tecidos de outros animais (HARMEL, 2004).

A reação de bioluminescência, resultante da oxidação de uma riboflavina 5-fosfato e um aldeído de longa cadeia na presença de oxigênio, é mediada pela enzima luciferase. Considerando que parte das funções vitais das bactérias estão relacionadas com os fatores ambientais, qualquer alteração no metabolismo celular dessas será representada pela diminuição da intensidade de luz.

O objetivo deste trabalho foi determinar a toxicidade dos principais herbicidas e inseticidas utilizados na lavoura de arroz irrigado, mediante ensaios de inibição de luminescência, usando como organismo-teste a bactéria luminescente Vibrio fischeri.

\section{MATERIAL E MÉTODOS}

Todos os procedimentos seguiram a metodologia apresentada pela norma ISO 11348-1 (1997).

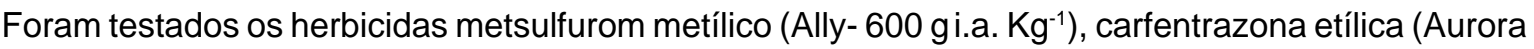
400 CE- 400 g i.a. L-1 $)$, bentazona (Basagran 600- 600 g i.a. $\mathrm{L}^{-1}$ ), quincloraque (Facet PM- 500 g i.a. $\mathrm{Kg}^{-1}$ ), oxifluorfem (Goal BR- 240 g i.a. $\mathrm{L}^{-1}$ ), bispiribaque-sódico (Nominee $400 \mathrm{SC}-400 \mathrm{~g}$ i.a. L $\mathrm{L}^{-1}$ ), pirazossulfurom etílico (Sírius- 250 g i.a. L L) , 2,4-D (DMA 806 BR- dimetilamida, 670 g i.a. L-1 $)$ e os 
inseticidas betaciflutrim (Bulldock 125 SC- 125 mg i.a. L-1), carbofuran (Furadan $50 \mathrm{G}-50$ g i.a. $\mathrm{Kg}^{-1}$ ), lambdacialotrina (Karatê 50 EC- 50 g i.a. $\mathrm{L}^{-1}$ ) e fipronil (Standak $250 \mathrm{FS}-250 \mathrm{~g}$ i.a. $\mathrm{L}^{-1}$ ). Para avaliar a sensibilidade do organismo, ao longo do período de realização dos ensaios, foram efetuados testes com a substância de referência Sulfato de Zinco $\left(\mathrm{ZnSO}_{4} \cdot 7 \mathrm{H}_{2} \mathrm{O}\right)$.

Usou-se o pacote LUMISTOX que consta de luminômetro para a leitura da intensidade luminosa, emitida pelas bactérias, e termobloco para a manutenção da temperatura nas cubetas durante o ensaio (STRAMOSK, 2004).

Em cada ensaio foram testadas 9 concentrações de cada produto, sendo a concentração máxima de 100 mg/L. Paralelamente, manteve-se o controle contendo somente solução salina a 20\%. Para cada nível de diluição e para o controle foram mantidas 2 réplicas para posterior exposição das bactérias às diluições correspondentes da solução-teste.

Os ensaios consistiam primeiramente na reativação das fotobactérias que foram distribuídas em alíquotas de $0,5 \mathrm{~mL}$ para as cubetas de leitura, efetuando-se após o período de incubação a leitura inicial de luminescência. Adicionou-se o mesmo volume de solução-teste às cubetas e após 30 minutos de exposição ao agente tóxico foram determinados os valores de luminescência finais.

$\mathrm{Na}$ avaliação do efeito inibitório dos produtos testados usou-se o programa LUMIS soft 4 (LANGE, 2000) para a determinação dos valores de $\mathrm{CE}_{20}$ e $\mathrm{CE}_{50}$ a partir das porcentagens de inibição de luminescência após 30 minutos de exposição.

Os índices de segurança foram estimados a partir dos valores da $\mathrm{CE}_{20}$ para cada ingrediente ativo e suas concentrações no ambiente com base nas doses recomendadas pelo fabricante para a cultura de arroz, considerando lâmina d'água de $10 \mathrm{~cm}$.

Empregou-se a análise de risco determinístico, proposta por SOLOMON (1997), que considera produtos com potencial risco de impacto ambiental aqueles que apresentam índices de segurança menores que 20.

\section{RESULTADOS E DISCUSSÃO}

\subsection{CARTA CONTROLE DE SENSIBILIDADE}

Os valores de inibição da luminescência obtidos nos ensaios com a substância de referência $\left(\mathrm{ZnSO}_{4} \cdot 7 \mathrm{H}_{2} \mathrm{O}\right.$ a $\left.25 \mathrm{mg} / \mathrm{L}\right)$ estão representados na Figura 1 . Apesar da variação dos valores, as porcentagens de inibição mantiveram-se entre 20 e $80 \%$, portanto, enquadraram-se no critério exigido para a validação dos ensaios, segundo a norma ISO 11348-1 (1997).

\section{FIGURA 1 - PORCENTAGEM DE INIBIÇÃO DA LUMINESCÊNCIA FRENTE À SUBSTÂNCIA DE REFERÊNCIA SULFATO DE ZINCO A 25 mg/L AO LONGO DOS MESES DE REALIZAÇÃO DOS ENSAIOS}

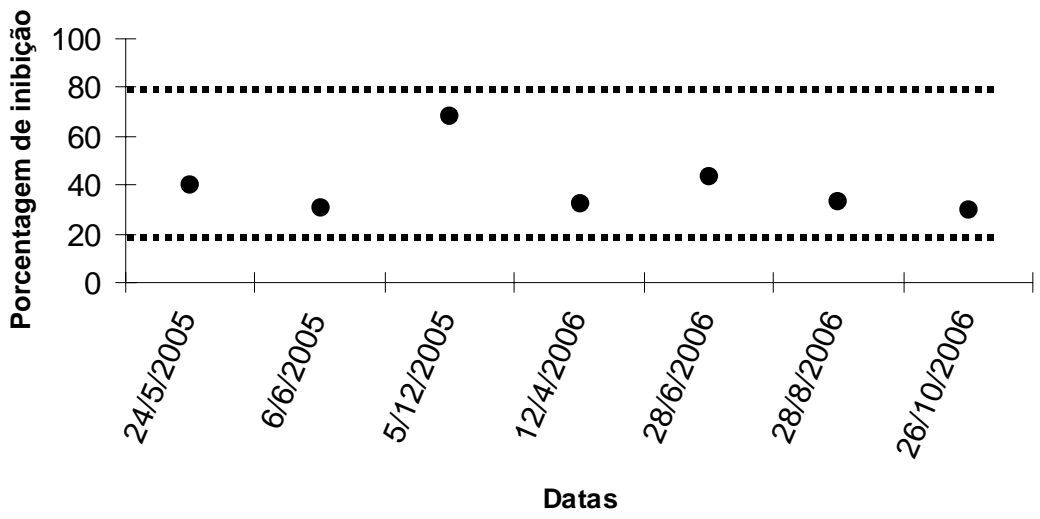


Os valores de porcentagem de inibição encontrados nas concentrações máximas testadas nos ensaios estão representados na Figura 2.

Os herbicidas Ally e Sírius e os inseticidas Standak e Bulldock mostraram valores de inibição inferiores a $20 \%$, não apresentando, portanto, efeito tóxico significativo sobre as bactérias. Altos percentuais de inibição da luminescência, próximo a 100\%, foram encontrados nos ensaios com os herbicidas 2,4-D, Aurora, Goal e com o inseticida Karatê. Foram observados valores intermediários de inibição para os herbicidas Basagran, Facet e Nominee, e o inseticida Furadan, quando comparados com os valores extremos atingidos nos ensaios com os demais produtos.

\title{
FIGURA 2 - VALORES DE PORCENTAGEM DE INIBIÇÃO DOS AGROQUÍMICOS TESTADOS NA CONCENTRAÇÃO MÁXIMA DE 100 mg/L (I.A.)
}

\author{
Teste de sensibilidade
}

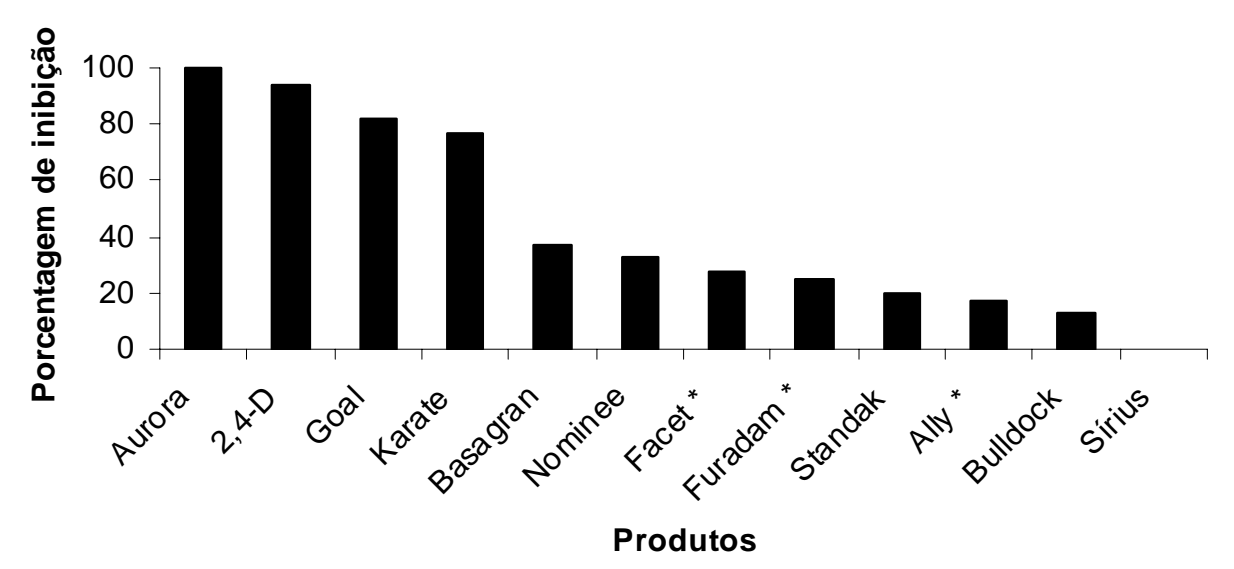

*Produtos testados a $100 \mathrm{mg} / \mathrm{L}$ do produto comercial, sendo facet: $50 \mathrm{mg} / \mathrm{L}$, furadan: $5 \mathrm{mg} / \mathrm{L}$ e ally: $60 \mathrm{mg} / \mathrm{L}$ para i.a.

$\mathrm{Na}$ Tabela 1 estão representados os valores de $\mathrm{CE}_{20}$ e $\mathrm{CE}_{50}$ para os produtos comerciais, ingredientes ativos e os índices de segurança determinados. Os produtos foram ordenados pelo risco em função do índice de segurança. Produtos com índices de segurança superiores a 20 não apresentam riscos segundo o critério de análise de risco proposto por SOLOMON (1997). Os herbicidas Ally, Nominee, Facet, Sírius e Basagran e os inseticidas Standak, Bulldock e Karatê não apresentam risco de impacto sobre o organismo-teste, ou mais especificamente sobre decompositores com base nas concentrações recomendadas pelo fabricante.

Segundo o USEPA (1999), os limites de classificação variam de acordo com o tipo de organismoteste e duração do experimento. Como o índice de SOLOMON (1997) foi elaborado em base dos valores de $\mathrm{CL}_{50} / \mathrm{CE}_{50}$ e não $\mathrm{CL}_{20} / \mathrm{CE}_{20}$ conforme apresentado neste trabalho, o critério 20 utilizado para classificar o risco do produto pode ser reduzido para 10 devido ao uso de $\mathrm{CE}_{20}$. Sob esse novo limite, os produtos com risco de impacto são Furadan, 2,4-D e Aurora.

Apenas os valores de $\mathrm{CE}_{20}$ ou $\mathrm{CE}_{50}$ não são suficientes para classificar determinada substância quanto ao grau de periculosidade, devendo-se considerar também a razão entre os valores de toxicidade e a provável concentração do produto no sistema. Sendo assim, ao contrário do que foi apresentado na Figura 2, o inseticida Karatê apresenta-se como produto seguro e o Furadan como produto de risco.

Em comparação com estudos realizados com outros organismos-teste (RESGALLA JR. et al., 2002; NAKAGOME et al., 2006; NAKAGOME, 2004), a bactéria Vibrio fischeri e o teste de bioluminescência apresentaram-se como ferramenta menos sensível na determinação dos efeitos tóxicos frente aos agroquímicos. Foram mais sensíveis somente para o herbicida 2,4-D em relação ao 
cladócero $D$. magna, entretanto, existe carência de informações para outros organismos-teste sob efeito desse produto. Para o herbicida Aurora e o inseticida Furadan, a bactéria mostrou sensibilidade similar a de outros organismos-teste.

\section{TABELA 1 - VALORES DE $C_{20} E_{2}$ CE $_{50} 30$ MINUTOS PARA OS PRODUTOS COMERCIAIS (P.C.) E INGREDIENTES ATIVOS (I.A.) DOS AGROQUÍMICOS SOBRE A BACTÉRIA LUMINESCENTE Vibrio fischeri, CONCENTRAÇÃO ESTIMADA, ÍNDICE DE SEGURANÇA, MEIA-VIDA NO SOLO, CONSTANTE DE PARTIÇÃO OCTANO-ÁGUA (Kow) E CLASSE TOXICOLÓGICA}

\begin{tabular}{|c|c|c|c|c|c|c|c|c|c|}
\hline \multirow[b]{2}{*}{ Produtos } & \multicolumn{2}{|c|}{$\mathrm{CE}_{20}(\mathrm{mg} / \mathrm{L})$} & \multicolumn{2}{|c|}{$\mathrm{CE}_{50}(\mathrm{mg} / \mathrm{L})$} & \multirow{2}{*}{$\begin{array}{l}\text { Concentraçã } \\
\text { o estimada } \\
(\mathrm{mg} / \mathrm{L})^{1}\end{array}$} & \multirow[b]{2}{*}{$\begin{array}{l}\text { Índice de } \\
\text { segurança }\end{array}$} & \multirow{2}{*}{ Kow $^{1}$} & \multirow[b]{2}{*}{$\begin{array}{l}\text { Meia- } \\
\text { vida no } \\
\text { solo }^{1}\end{array}$} & \multirow[b]{2}{*}{$\begin{array}{c}\text { Classe } \\
\text { Toxicológica }^{5}\end{array}$} \\
\hline & $\begin{array}{l}\mathrm{CE}_{20} \\
\text { (p.c.) }\end{array}$ & $\begin{array}{l}\mathrm{CE}_{20} \\
\text { (i.a.) }\end{array}$ & $\begin{array}{l}\mathrm{CE}_{50} \\
\text { (p.c.) }\end{array}$ & $\begin{array}{l}\mathrm{CE}_{50} \\
\text { (i.a.) }\end{array}$ & & & & & \\
\hline Furadam & 64,79 & 3,23 & $>100$ & $>5$ & 1 & $3,23^{*}$ & $114-232^{3}$ & $\begin{array}{l}\text { 2-110 } \\
\text { dias }^{4}\end{array}$ & 1 \\
\hline $2,4-D$ & 0,005 & 3,87 & 0,020 & 13,90 & 0,72 & $5,38^{\star}$ & - & - & 1 \\
\hline Aurora & 0,0008 & 0,35 & 0,002 & 1,02 & 0,06 & $5,83^{\star}$ & - & - & II \\
\hline Goal & 0,015 & 3,83 & 0,080 & 19,23 & 0,36 & $10,60^{*}$ & 29.400 & $\begin{array}{l}\text { 30-40 } \\
\text { dias }\end{array}$ & II \\
\hline Karatê & 0,023 & 1,15 & 0,2 & 10 & 0,05 & 23,00 & - & - & II \\
\hline Basagran & 0,068 & 40,87 & $>0,16$ & $>100$ & 0,96 & 42,58 & $0,35^{4}$ & - & III \\
\hline Facet & 72 & 36 & $>100$ & $>50$ & 0,375 & 96,00 & 0,07 & - & III \\
\hline Nominee & 0,114 & 45,83 & $>0,25$ & $>100$ & 0,05 & 916,60 & - & $\begin{array}{c}60 \\
\text { dias }\end{array}$ & II \\
\hline Standak & $>0,4$ & $>100$ & $>0,4$ & $>100$ & 0,04 & $>2.500,00$ & - & - & IV \\
\hline Sírius & 0,4 & $>100$ & 0,4 & $>100$ & 0,02 & $>5.000,00$ & - & $\begin{array}{r}7-15 \\
\text { dias } \\
\end{array}$ & IV \\
\hline Bulldock & $>0,8$ & $>100$ & $>0,8$ & $>100$ & 0,006 & $>16.000,00$ & - & - & II \\
\hline Ally & $>100$ & $>60$ & $>100$ & $>60$ & 0,002 & $>30.000,00$ & 1,0 & $\begin{array}{c}\text { 30-120 } \\
\text { dias }\end{array}$ & III \\
\hline
\end{tabular}

${ }^{1}$ RODRIGUES e ALMEIDA (1998); ${ }^{2}$ Índice de segurança = $\mathrm{CE}_{20}$ (i.a.)/concentração estimada (SOLOMON, 1997); ${ }^{3} \mathrm{FRELLO}$ (1998); ${ }^{4}$ EMBRAPA (2006); ${ }^{5}$ IRGA, 2002.

Os lipopolissacarídeos da membrana externa da bactéria Vibrio fischeri a protegem contra certas moléculas tóxicas (PELCZAR, CHAN e KRIEG, 1996). Esse fato poderia explicar a baixa sensibilidade dessa bactéria frente à maioria dos compostos testados. Porém, os diferentes modos de ação e características químicas dos produtos podem interagir de maneiras distintas com o organismo e o sistema-teste.

Na Tabela 3 estão expostos os mecanismos de ação dos herbicidas testados e o respectivo efeito tóxico sobre a bactéria luminescente Vibrio fischeri. Entre os produtos que apresentaram toxicidade estão aqueles que atuam sobre enzimas, especialmente oxidases. Os herbicidas Aurora e Goal atuam como inibidores da protoporfirinogênio oxidase e considerando que a luciferase (enzima chave no processo de bioluminescência) também é uma oxidase, a toxicidade elevada desses produtos sobre a bactéria pode ser explicada pela sua ação específica sobre essa classe de enzimas.

Os herbicidas inibidores da enzima acetolactato sintase (ALS) apresentaram baixa ou nenhuma toxicidade para Vibrio fischeri, fato que pode ser explicado pela possível seletividade da membrana bacteriana a esses compostos. 
Os herbicidas 2,4-D, Basagran e Facet atuam sobre funções fisiológicas específicas de vegetais, sendo assim, sua ação tóxica sobre a bactéria confirma a baixa especificidade e seletividade desses produtos.

\section{TABELA 2 - MECANISMO DE AÇÃO DOS HERBICIDAS TESTADOS E SEU EFEITO TÓXICO SOBRE A BACTÉRIA LUMINESCENTE Vibrio fischeri}

\begin{tabular}{ccc}
\hline Produtos & Modo de ação & Efeito tóxico para Vibrio fischeri \\
\hline 2,4-D & $\begin{array}{c}\text { Mimetizador da auxina } \\
\text { Inibidor da enzima acetolactato } \\
\text { síntase (ALS) } \\
\text { Inibidor da enzima }\end{array}$ & Toxicidade elevada \\
Aurora (Carfentrazona-etílica) & $\begin{array}{c}\text { Sem efeito } \\
\text { protoporfirinogênio oxidase } \\
\text { Inibidor do fotossistema II }\end{array}$ & Toxicidade elevada \\
Basagran (Bentazona) & $\begin{array}{c}\text { Mimetizador da auxina } \\
\text { Inibidor da enzima }\end{array}$ & Toxicidade baixa \\
Facet (Quincloraque) & $\begin{array}{c}\text { Toxicidade baixa } \\
\text { Goal (Oxifluorfem) }\end{array}$ & Toxicidade elevada \\
Nominee (Bispiribaque-sódico) & $\begin{array}{c}\text { Inibidor da enzima acetolactato } \\
\text { síntase (ALS) }\end{array}$ & Toxicidade baixa \\
Sírius (Pirazossulfurom-etílico) & $\begin{array}{c}\text { Inibidor da enzima acetolactato } \\
\text { síntase (ALS) }\end{array}$ & Sem efeito \\
\hline
\end{tabular}

A avaliação de estudos sobre o comportamento dos agroquímicos no ambiente em conjunto com resultados obtidos em bioensaios, realizados com diferentes organismos-teste, assume extrema importância para a criteriosa análise do risco potencial de impacto desses compostos sobre o meio ambiente (RESGALLA JR. et al., 2007).

\section{CONCLUSÃO}

Os testes de bioluminescência com a bactéria Vibrio fischeri apresentaram baixa sensibilidade para a maioria dos agroquímicos utilizados na cultura do arroz irrigado. O teste de bioluminescência mostrou sensibilidade similar a de outros organismos-teste para os produtos Furadan, 2,4-D e Aurora, os únicos que apresentaram risco de impacto ambiental.

\section{ABSTRACT \\ ACUTE TOXICITY OF THE MAIN HERBICIDES AND INSECTICIDES USED IN THE IRRIGATED RICE CROP ON THE BACTERIUM Vibrio fischeri}

Santa Catarina and Rio Grande do Sul are the largest irrigated rice producing states in Brazil, and are highlighted for their intensive use of agricultural chemicals for the control of undesireable competitors and consumers of the crop. In order to evaluate the toxic potential of these agricultural chemicals, and their effects on non-target organisms, this work sought to determine the $\mathrm{EC}_{20}$ and $\mathrm{EC}_{50}$ of the main herbicides and insecticides used in the rice crop, using as a test organism the bioluminescent bacterium Vibrio fischeri. Short duration static tests were carried out (30 minutes), based on the inhibition of luminescence of the bacteria after exposure to the toxic agent. The products tested were the herbicides 2,4-D (DMA 806 BRdimethylamide), metsulfuron-methyl (Ally), carfentrazone-ethyl (Aurora), bentazon (Basagran), quinclorac (Facet), oxyfluorfen (Goal), bispyribac-sodium (Nominee) and pyrazosulfuron-ethyl (Sírius), and the 
insecticides betacyflutrin (Bulldock), carbofuran (Furadan), lambdacyhalothrin (Karate) and fipronil (Standak) at maximum concentrations of $100 \mathrm{mg} / \mathrm{L}$ (a.i.). The herbicides Ally and Sírius and the insecticides Bulldock and Standak did not present toxicity for the bacterium Vibrio fischeri, as they resulted in an inhibitory effect of less than $20 \%$. Basagram, Facet, Nominee and Furadam presented toxicity with a $\mathrm{EC}_{20}$ (a.i.) of $40.87 \mathrm{mg} / \mathrm{L}, 36.00 \mathrm{mg} / \mathrm{L}, 45.83 \mathrm{mg} / \mathrm{L}$ and $3.23 \mathrm{mg} / \mathrm{L}$, respectively, and for the products 2.4-D, Aurora, Goal and Karate, the $\mathrm{EC}_{50}$ values for the active ingredient obtained were $13.90 \mathrm{mg} / \mathrm{L}, 1.02 \mathrm{mg} / \mathrm{L}, 19.23 \mathrm{mg} / \mathrm{L}$ and $10 \mathrm{mg} / \mathrm{L}$, respectively. Compared with studied carried out with other test organisms, the bacterium $\mathrm{V}$. fischeri and the bioluminescence test presented low sensitivity to the toxicity of the agricultural chemicals.

KEY-WORDS: TOXICITY TESTS; HERBICIDES; INSECTICIDES; BIOLUMINESCENCE; Vibrio fischeri.

\section{REFERÊNCIAS}

1 ALBA, A.R.F.; GUIL, L.H.; LÓPEZ, G.D.; CHISTI, Y. Toxicity of pesticide in wastewater: a comparative assessment of rapid bioassays. Analytica Chimica Acta, v. 426, n. 2, p. 289-301, 2001.

2 DESCHAMPS, F.C.; NOLDIN, J.A.; EBERHARDT, D.S.; HERMES, L.C.; KNOBLAUCH, R. Resíduos de agroquímicos em águas nas áreas de arroz irrigado, em Santa Catarina. In: CONGRESSO BRASILEIRO DE ARROZ IRRIGADO, 3., E REUNIÃO DA CULTURA DO ARROZ IRRIGADO, 25., 2003, Balneário Camboriú. Anais... Itajaí: Epagri, 2003. p. 683685.

3 EMBRAPA. Principais herbicidas recomendados para cultura de soja no preparo convencional e no sistema plantio direto. Disponível em: http://www.cnpt.embrapa.br/biblio/do/p_do62_17.htm. Acesso em: 26 nov. 2006.

4 EPA. Environmental Protection Agency. ECOFRAM. Aquatic Report. Washington D.C., 1999.

5 FRELLO, C.P. Avaliação da toxicidade aguda do agrotóxico carbofuran utilizando reativos biológicos: Poecila reticulata e Daphnia magna. Florianópolis, 1998. 96 p. Dissertação (Mestrado em Engenharia Ambiental), Departamento de Engenharia Sanitária e Ambiental, Universidade Federal de Santa Catarina.

6 HARMEL, V.C. Padronização de um teste de toxicidade crônica com a bactéria luminescente Vibrio fischeri para a análise de qualidade de águas superficiais. Blumenau, 2004. 100 f. Dissertação (Mestrado em Engenharia Ambiental), Centro de Ciências Tecnológicas, Universidade Regional de Blumenau.

7 IRGA. Instituto Rio Grandense do Arroz. Acompanhamento semanal da colheita. Disponível em: <http:// www.irga.rs.gov.br>. Acesso em: 2 junho 2002.

8 ISO. International Organization For Standardization. ISO 11348-1: water quality - determination of the inhibitory effect of water samples on the light emission of Vibrio fischeri (Luminecescentbacteria test). Geneve,1997.

9 LANGE, Bruno. GmbH: LUMIsoft 4. Ver 1.001. [S.L.], 2000.

10 NAKAGOME, F.K. Toxicidade aguda dos principais herbicidas e inseticidas utilizados na lavoura do arroz irrigado sobre o cladócera Daphnia magna e o paulistinha Danio rerio. Itajaí, 2004. 65 f. Monografia (Graduação em Oceanografia), Centro de Ciências Tecnológicas da Terra e do Mar, Universidade do Vale do Itajaí.

11 NAKAGOME, F.K.; NOLDIN, J.A.; RESGALLA JR., C. Toxicidade aguda e análise de risco de herbicidas e inseticidas utilizados na lavoura do arroz irrigado sobre o cladócero Daphnia magna. Pesticidas: revista de ecotoxicologia e meio ambiente, Curitiba, v. 16, p. 93-100, 2006.

12 NOLDIN, J.A. Sistema de produção orgânica de arroz irrigado. Itajaí: FUNDAGRO-EPAGRI, 2005. 55 p. (FUNDAGRO-Agricultura Familiar). Projeto em andamento.

13 NOLDIN, J.A. et al. Estratégia de coleta de amostras de água para monitoramento do impacto ambiental da cultura do arroz irrigado. In: CONGRESSO BRASILEIRO DEARROZ IRRIGADO, 2., E REUNIÃO DA CULTURA DO ARROZ IRRIGADO, 24., 2001, Porto Alegre. Anais... Porto Alegre: IRGA, EMBRAPA, EPAGRI e SOSBAI, 2001. p. 760-762.

14 PELCZAR JR., M. J.; CHAN, E. C. S.; KRIEG, N. R. Microbiologia: conceitos e aplicações. São Paulo: Makron Books do Brasil, 1996.

15 RESGALLA JR., C.; NOLDIN, J.A.; SANTOS, A.L.; SATO, G.; EBERHARDT, D.S. Toxicidade aguda de herbicidas e inseticida utilizados na cultura do arroz irrigado sobre juvenis de carpa (Cyprinus carpio). Pesticidas: revista ecotoxicologia e meio ambiente, Curitiba, v. 12, p. 59-68, 2002.

16 RESGALLA JR., C.; NOLDIN, J.A.; TAMANAHA, M.; DESCHAMPS, F.C.; EBERHARDT, D.; RÖRIG, L.R. Risk analysis of herbicide quinclorac residues in irrigated rice areas, Santa Catarina, Brazil. Ecotoxicology, v. 16, p. 565-571, 2007. 
17 RODRIGUES, B.N.; ALMEIDA, F.S. Guia de herbicidas. 4. ed. Londrina: Editora. dos Autores, 1998. 648 p.

18 SOLOMON, K.R. Advances in the evaluation of the toxicological risks of herbicides to the environment. In: CONGRESSO BRASILEIRO DA CIÊNCIA DAS PLANTAS DANINHAS, 21., Caxambu. Anais...Caxambu, MG: SBCPD, 1997. p.163-172.

19 STRAMOSK, C.A. Uso de ensaios de biotoxicidade com bactérias luminescentes (LUMISTOX) para a avaliação da qualidade da água do Rio Itajaí-Açú- SC. Itajaí, 2004. 47 f. Monografia (Graduação em Oceanografia), Centro de Ciências Tecnológicas da Terra e do Mar, Universidade do Vale do Itajaí.

20 TAMANAHA, M.S.; RESGALLA JR., C.; RÖRIG, L.R.; CHACÓN, P.Z.; NOLDIN, J.A.; EBERHARDT, D.S.; BEAUMORD, A.C. Avaliação da toxicidade de seis agroquímicos utilizados na cultura de arroz irrigado em teste de inibição de crescimento algal. In: CONGRESSO BRASILEIRO DE ARROZ IRRIGADO, 3., E REUNIÃO DA CULTURA DE ARROZ IRRIGADO, 25., 2003, Balneário Camboriú. Anais... Itajaí: Epagri, 2003. p. 714-716. 